\title{
Squeeze-film effects in MEMS devices with perforated plates for small amplitude vibration
}

\author{
C. Feng $\cdot$ Ya-Pu Zhao $\cdot$ D. Q. Liu
}

Received: 3 June 2006/ Accepted: 4 October 2006/Published online: 15 November 2006

(C) Springer-Verlag 2006

\begin{abstract}
Squeeze-film effects of perforated plates for small amplitude vibration are analyzed through modified Reynolds equation (MRE). The analytical analysis reckons in most important influential factors: compressibility of the air, border effects, and the resistance caused by vertical air flow passing through perforated holes. It is found that consideration of air compressibility is necessary for high operating frequency and small ratio of the plate width to the attenuation length. The analytical results presented in this paper agree with ANSYS simulation results better than that under the air incompressibility assumption. The analytical analysis can be used to estimate the squeeze-film effects causing damping and stiffness added to the system. Since the value of Reynolds number involved in this paper is low $(<1)$, inertial effects are neglected.
\end{abstract}

\section{Introduction}

For microelectromechanical systems (MEMS) devices, if two nearby planar structures have relative motion in their normal direction, the thin layer of air between

\section{Feng · Y.-P. Zhao $(\bowtie)$}

State Key Laboratory of Nonlinear Mechanics (LNM), Institute of Mechanics, Chinese Academy of Sciences, Beijing 100080, China

e-mail: yzhao@lnm.imech.ac.cn

C. Feng · D. Q. Liu

Department of Mathematics and Mechanics, University of Science and Technology Beijing,

Beijing 100083, China them will produce squeeze-film effects, which can alter the dynamic response of MEMS devices by adding stiffness and damping to the system. To reduce squeeze-film effects, perforating holes in microstructures are widely used in MEMS devices (Davies 1997). Actually, the perforations in microstructures have several functions as mentioned in (Andrews et al. 1993; Homentcovschi and Miles 2004; Starr 1996): (1) reducing squeeze-film effects; (2) controlling the dynamic response of MEMS devices; (3) enhancing the etching rate of sacrificial layers in the micro-fabrication process.

Perforating holes in microstructures can reduce the squeeze-film effects caused by horizontal air flow, but the air flow escaping through perforated holes adds new viscous resistance. Therefore, the conventional Reynolds equation for determining squeeze-film effects of non-perforated planar structures is no longer applicable.

A lot of efforts have been devoted to seek ways to determine squeeze-film effects of perforated planar structures. Under the assumption that air is incompressible, some papers gave analytical expressions and numerical analysis to evaluate the squeeze-film damping. Škov (1967) derived an expression to determine the squeeze-film damping of perforated plates in some MEMS devices. The derivation didn't consider the air compressibility and the resistance offered by vertical air flow passing through holes. It is reasonable for low operating frequency and large ratio of the hole size to the thickness of the perforated planar structure. By adding a penetrating term to fluid continuity equation, Bao et al. (2003) developed a modified Reynolds equation (MRE) to determine the squeeze-film damping caused by both horizontal and 
vertical air flow. Besides analytical analysis, some other methods like finite element method (FEM) (Kim et al. 1999; Schrag and Wachutka 2004; Yan and Lal 2006; Yang et al. 1997) and finite difference method (Veijola and Mattila 2001; Veijola et al. 2001) were also used and developed to estimate the squeeze-film damping. FEM is considered to be an effective way to evaluate squeeze-film damping. However, FEM is time consuming for large-scale structures and it can't give much suggestion to device design optimization.

Recent work by Homentcovschi and Miles (2004) and Mohite et al. (2005) gave analytical approximations and simulations to the squeeze-film effects of perforated plates. Homentcovschi considered the perforated plate as infinite, and divided the plate into mass pressure cells. The analysis was made for one pressure cell, which considered the air compressibility, inertial effects and possible slip of the gas on the plate. Then the total squeeze-film effects for the whole plate can be obtained by multiplication. Besides, the paper also gave analysis on the caused error while dividing the plate into circular cells. Mohite introduced a parameter called diffusion factor and presented discussions on the damping and spring effects by observing the pattern of ANSYS simulation. Since the ratio of the hole radius to the hole depth involved in the paper is small, the analysis didn't consider the resistance offered by vertical air flow. Homentcovschi and Mohite both considered the perforated plate as infinite and did investigations on one cell to obtain the squeeze-film effects for the whole plate by multiplication. So their analysis neglected border effects. As a matter of fact, the plates are always finite, and squeeze-film effects depend on the plates' boundary. So for a better approximation of squeeze-film effects, border effects should be considered.

In this paper, a commonly used rectangular perforated plate is considered, and another kind of MRE considering air compressibility, border effects and the resistance offered by vertical air flow is obtained to evaluate squeeze-film effects. As FEM is an effective way to evaluate squeeze-film effects of small-scale structures, simulations by ANSYS are made for a typical one-dimensional rectangular perforated plate to do comparisons between different analytical results and simulation results.

Since the value of Reynolds number involved in this paper is low, inertial effects are neglected. However, for devices with larger air gap and high operating frequency, inertial effects will be no longer neglectable. A new way or model has to be developed.

\section{Modified Reynolds equation for squeeze-film effects of finite, perforated plate}

2.1 Conventional Reynolds equation for squeezefilm effects of non-perforated plate

If the two nearby plates having relative motion in their normal direction are non-perforated, squeeze-film effects can be evaluated by the conventional Reynolds equation (Bao 2000; Starr 1996)

$\frac{\partial}{\partial x}\left(\rho \frac{d^{3}}{\mu} \frac{\partial p}{\partial x}\right)+\frac{\partial}{\partial y}\left(\rho \frac{d^{3}}{\mu} \frac{\partial p}{\partial y}\right)=12 \frac{\partial(\rho d)}{\partial t}$,

where $p=p(x, y)$ is the pressure distribution of the squeeze-film, $\rho$ the density of air, $d$ the gap between the two nearby plates, and $\mu$ the viscosity of air. Since the air gap between the two nearby plates is always small in MEMS devices, the viscosity of air, $\mu$, should be substituted by the effective viscosity (Fukui and Kaneko 1988), $\mu_{\text {eff }}=\frac{\mu}{1+9.638 K^{1.159}}$, to consider the rarefaction of the air, where $K_{n}^{n}$ is the Knudsen number defined as the ratio of the molecular mean free path length to a representative physical length scale.

\subsection{Modified Reynolds equation and linearized form for squeeze-film effects of perforated plate}

As mentioned in the introduction section, microstructures are commonly perforated to reduce squeeze-film effects, and the conventional Reynolds equation is no longer applicable for perforated plates because of the additional air flow passing through perforated holes. However, the conventional equation can be modified to be applicable for perforated plate. As most papers did (Bao et al. 2003; Homentcovschi and Miles 2004; Mohite et al. 2005), the uniformly perforated plate with mass holes can be divided into cells. As shown in Fig. $1, R_{i}$ and $R_{o}$ are the inner and outer radii of the

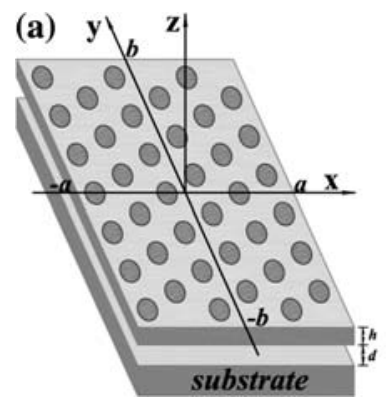

(b)

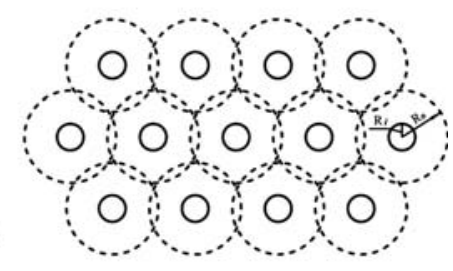

Fig. 1 Schematic structure of a rectangular perforated plate. a Geometries of the rectangular plate. b Part of the divided plate 
divided pressure cell, respectively. As the divided pressure cell is much smaller than the plate, the pressure under the plate can be considered as a continuous distribution. And the air flow passing through a hole can be considered as penetrating out through the whole divided pressure cell. Then the fluid continuity equation for perforated plate can be modified as (Bao et al. 2003)

$\frac{\partial\left(\rho q_{x}\right)}{\partial x}+\frac{\partial\left(\rho q_{y}\right)}{\partial y}+\rho Q_{z}+\frac{\partial(\rho d)}{\partial t}=0$.

For isothermal flow we use the condition $p \propto \rho$, and Eq. 2 becomes

$\frac{\partial\left(p q_{x}\right)}{\partial x}+\frac{\partial\left(p q_{y}\right)}{\partial y}+p Q_{z}+\frac{\partial(p d)}{\partial t}=0$,

where $q_{x}=-\frac{d^{3}}{12 \mu_{\text {eff }}} \frac{\partial p}{\partial x}, q_{y}=-\frac{d^{3}}{12 \mu_{\text {eff }}} \frac{\partial p}{\partial y}$, and $Q_{z}$ is the socalled penetrating rate which is the average volume passing through holes for a unit area per unit time. Since the divided cell is much smaller than the plate, the penetrating air flow for every cell can be considered as autonomous. In the following, analysis is made for one cell to derive the expression of $Q_{z}$.

The penetrating air flow passing through holes is caused by the pressure difference between the ambient and the squeeze-film, and the total pressure difference for one cell is composed of two parts, $p_{\mathrm{h}}$ and $p_{\mathrm{v}}$, which are caused by horizontal and vertical air flows, respectively.

According to the derivation in the paper by Gabrielson (1993) the pressure difference, $p_{\mathrm{h}}$, which is caused by the horizontal air flow from the periphery to the centre of a pressure cell, can be approximately as

$p_{\mathrm{h}}=\frac{3 \mu_{\mathrm{eff}} R_{0}^{2}}{2 d^{3}} K(\beta) V$

where $V$ is the volume of air passing through the hole per unit time, $\beta=R_{\mathrm{i}} / R_{0}$. and $K(\beta)=4 \beta^{2}-\beta^{2}-4 \ln \beta-3$.

According to the law of Hagen-Poiseuille flow, the pressure difference, $p_{\mathrm{v}}$, which is caused by the vertical air flow from one end to the other end of the hole, is

$p_{\mathrm{v}}=\frac{8 \mu_{\mathrm{eff}} h}{\pi R_{\mathrm{i}}^{4}} V$

where $h$ is the thickness of the plate. For most perforated microstructures in which the inner radius of the hole, $R_{\mathrm{i}}$, is comparable with the plate thickness, air flow in the hole is not a fully developed Poiseuille flow. So an effective plate thickness is introduced to include the non-fully developed effects. The effective plate thickness can be obtained from the modified Poiseuille equation by Sharipov and Seleznev (1997)

$p_{\mathrm{v}}=\frac{8 \mu_{\mathrm{eff}}\left(h+3 \pi R_{\mathrm{i}} / 8\right)}{\pi R_{\mathrm{i}}^{4}} V=\frac{8 \mu_{\mathrm{eff}} h_{\mathrm{eff}}}{\pi R_{\mathrm{i}}^{4}} V$,

where, $h_{\mathrm{eff}}=h+\frac{3 \pi R_{\mathrm{i}}}{8}$, is the effective plate thickness.

Therefore, the total pressure difference is

$p-p_{\mathrm{a}}=p_{\mathrm{h}}+p_{\mathrm{v}}=\left[1+\frac{3 R_{\mathrm{i}}^{4} K(\beta)}{16 h_{\mathrm{eff}} d^{3}}\right] p_{\mathrm{v}}=\eta(\beta) p_{\mathrm{v}}$,

where $p_{\mathrm{a}}$ is the ambient pressure and $\eta(\beta)=1+\frac{3 R_{i}^{4} K(\beta)}{16 h_{\mathrm{eff}} d^{3}}$. So the penetrating rate is

$Q_{Z}=\frac{V}{\pi R_{0}^{2}}=\frac{\beta^{2} R_{\mathrm{i}}^{2}}{8 \mu_{\mathrm{eff}} h_{\mathrm{eff}}} p_{\mathrm{v}}=\frac{\beta^{2} R_{\mathrm{i}}^{2}}{8 \mu_{\mathrm{eff}} h_{\mathrm{eff}}} \cdot \frac{p-p_{\mathrm{a}}}{\eta(\beta)}$.

Substituting Eq. 8 into Eq. 3, the modified fluid continuity equation can be recast to the MRE as

$\frac{\partial}{\partial x}\left(p d^{3} \frac{\partial p}{\partial x}\right)+\frac{\partial}{\partial y}\left(p d^{3} \frac{\partial p}{\partial y}\right)-\frac{p\left(p-p_{\mathrm{a}}\right)}{l^{2}}=12 \mu_{\mathrm{eff}} \frac{\partial(p d)}{\partial t}$,

with boundary conditions

$p( \pm a, y)=p_{\mathrm{a}}, p(x, \pm b)=p_{\mathrm{a}}$,

where $l=\sqrt{\frac{2 h_{\text {eff }} d^{3} \eta(\beta)}{3 \beta^{2} R_{\mathrm{i}}^{2}}}$ defined as attenuation length (Bao et al. 2003). The attenuation length $l$ reflects border effects. For the region $D \in(-a+l<x<$ $a-l \cap-b+l<y<b-l)$ under the plate, the pressure value can be considered as constant. But for the rest region from inner to the borders, the pressure drops significantly. The pressure drop near the borders is due to border effects and smaller attenuation length means weaker border effects.

During the above derivation, we assume that the plate is perforated with circular holes. In fact, square holes are also used in MEMS devices quite often. To include this situation, the air flow passing through a square hole with hole side $w$ can be estimated by the equation (Sharipov 1999)

$V=0.035 \frac{p_{\mathrm{v}} w^{4}}{\mu_{\mathrm{eff}} h_{\mathrm{eff}}}$.

So the equivalent inner radius for a square hole is approximated as

$R_{\text {ieq }}=0.547 w$. 
The following dimensionless quantities are defined to transform Eq. 9 into dimensionless form

$$
\left\{\begin{array}{c}
P=\frac{p}{p_{\mathrm{a}}}, X=\frac{x}{a}, Y=\frac{y}{a}, D=\frac{d}{d_{0}}, L=\frac{a}{l} \\
\tau=\omega t, A_{R}=\frac{b}{a}, \sigma=\frac{12 \mu_{\mathrm{eff}} \omega a^{2}}{p_{\mathrm{a}} d_{0}^{2}}=\hat{\sigma} L^{2}
\end{array},\right.
$$

where $a$ and $b$ are half width and half length of the plate, respectively, $\hat{\sigma}=\frac{12 \mu_{\mathrm{eff}} \omega l^{2}}{p_{\mathrm{a}} d_{0}^{2}}=\frac{\sigma}{L^{2}}, d_{0}$ is the initial gap between the two nearby plates, $\omega$ is operating frequency in radians per second, $\sigma$ is the squeeze number and $t$ is the time. Then Eq. 9 becomes

$$
\begin{gathered}
\frac{\partial}{\partial X}\left(P D^{3} \frac{\partial P}{\partial X}\right)+\frac{\partial}{\partial Y}\left(P D^{3} \frac{\partial P}{\partial Y}\right) \\
-L^{2} P(P-1)=\sigma \frac{\partial}{\partial \tau}(P D),
\end{gathered}
$$

with corresponding boundary conditions

$P( \pm 1, Y)=1, P\left(X, \pm A_{R}\right)=1$.

For MEMS devices, the vibrating amplitude is always much smaller than the air gap, and the pressure difference is much smaller than the ambient pressure. So the dimensionless pressure difference and amplitude can be approximated as

$P=1+\overline{\mathrm{P}}, \quad D=1+\varepsilon$,

$\bar{P}$ and $\varepsilon$ are both infinitesimal compared to 1 . To the lower order in $\bar{P}$ and $\varepsilon$, Eq. 14 becomes the linearized form as

$\nabla^{2} \overline{\mathrm{P}}-L^{2} \overline{\mathrm{P}}=\sigma\left(\frac{\partial \overline{\mathrm{P}}}{\partial \tau}+\frac{\partial \varepsilon}{\partial \tau}\right)$.

In the case of simple harmonic vibration, we have

$\overline{\mathrm{P}}=\overline{\mathrm{P}}_{0}(X, Y) \cdot e^{-j \tau}, \quad \varepsilon=\varepsilon_{0} \cdot e^{-j \tau}$,

where $\varepsilon_{0}$ is the dimensionless amplitude. Equation 17 then becomes

$\nabla^{2} \Psi_{0}-\left(L^{2}-j \sigma\right) \Psi_{0}=-L^{2} \varepsilon_{0}$,

with boundary conditions
$\Psi_{0}( \pm 1, Y)=\varepsilon_{0}, \quad \Psi_{0}\left(X, \pm A_{R}\right)=\varepsilon_{0}$.

If dimensionless pressure difference $\overline{\mathrm{P}}_{0}$ is obtained, dimensionless force $F$ caused by squeeze-film effects can be expressed as

$F=\overline{\mathrm{P}}_{0}(X, Y) \mathrm{d} X \mathrm{~d} Y \quad\left(-1 \leq X \leq 1,-A_{R} \leq Y \leq A_{R}\right)$.

The force, $F$, can be written as $\left(F=F_{\mathrm{R}}+j F_{\mathrm{I}}\right)$, where $F_{\mathrm{R}}$ is the spring force and $F_{\mathrm{I}}$ is the damping force. Hence, the stiffness and the damping added to the system by squeeze-film effects can be written as

$K=\frac{F_{\mathrm{R}}}{\varepsilon_{0}}, \quad C=\frac{F_{\mathrm{I}}}{\omega \varepsilon_{0}}$.

So in the following sections, we just give particular attention and discussions to the dimensionless pressure difference $\overline{\mathrm{P}}_{0}$.

\section{Theoretical analysis}

\subsection{One-dimensional perforated plate}

Here a one-dimensional rectangular perforated plate is first considered to reveal the characteristics of pressure distributions. The plate length is far larger than the width $(2 b \gg 2 a$ as shown in Fig. 1$)$, so the pressure caused by squeeze-film effects can be thought as a function of $X$. Then Eq. 19 is

$\frac{\mathrm{d}^{2}}{\mathrm{~d} X^{2}} \Psi_{1}(X)-\left(L^{2}-j \sigma\right) \Psi_{1}(X)=-L^{2} \varepsilon_{0}$

Using boundary condition of $\Psi_{1}( \pm 1)=\varepsilon_{0}$, the solution of Eq. 23 is found to be

$\Psi_{1}(X)=\overline{\mathrm{P}}_{1}(X)_{\mathrm{S}}+j \overline{\mathrm{P}}_{1}(X)_{\mathrm{D}}+\varepsilon_{0}$

where

$\overline{\mathrm{P}}_{1}(X)_{\mathrm{S}}=\frac{\sigma \varepsilon_{0}}{L^{4}+\sigma^{2}}\left[\left(A_{1}-1\right) \sigma+B_{1} L^{2}\right]$

$\overline{\mathrm{P}}_{1}(X)_{\mathrm{D}}=\frac{\sigma \varepsilon_{0}}{L^{4}+\sigma^{2}}\left[B_{1} \sigma-\left(A_{1}-1\right) L^{2}\right]$,

and 
The real part, $\overline{\mathrm{P}}_{1}(X)_{\mathrm{S}}$, is the spring pressure and the imaginary part, $\overline{\mathrm{P}}_{1}(X)_{\mathrm{D}}$, is the damping pressure.

When operating frequency is low or the value of $L$ is high, the value of squeeze number is much smaller than the value of $L$ and the air being compressed under the plate is little. By using the condition $\hat{\sigma}=\sigma / L^{2} \rightarrow 0$, spring pressure, $\overline{\mathrm{P}}_{1}(X)_{\mathrm{S}}$, tends to be zero and damping pressure, $\overline{\mathrm{P}}_{1}(X)_{\mathrm{D}}$, can be simplified as

$\overline{\mathrm{P}}_{1}(X)_{\mathrm{D}}=\frac{\sigma \varepsilon_{0}}{L^{2}}[1-\cosh (L X) / \cosh (L)]$.

Equation 28 is just the dimensionless form of the expression by Bao et al. (2003) which didn't consider the air compressibility. Comparing Eqs. 26, 28, it is found that in the case of air incompressibility, damping pressure is proportional to operating frequency, which can be expressed as $\overline{\mathrm{P}}_{\mathrm{D}} \propto f$. So damping pressure increases as operating frequency growing at all times. But in the case of air compressibility, the relation between damping pressure and operating frequency is not that simple. In the following, for a perforated plate with the same value of $L(L=5)$ but different vibrating frequencies, investigations are made on the distributions of spring and damping pressure in the cases of air incompressibility and compressibility, respectively.

From Figs. 2, 3, it can be found that (1) for low operating frequency, the discrepancy between the cases of air incompressibility and compressibility is small, but becomes larger for higher operating frequency; (2) spring pressure increases rapidly as operating frequency growing, and becomes comparable with damping pressure for high operating frequency.

Since $L$ is a comprehensive and important dimensionless quantity in MRE, which includes the information of the perforated plate and the air gap. In the following, with the same operating frequency, we will investigate the influence of $L$ on the squeeze-film caused pressure.

From Figs. 4, 5, it can be found that (1) spring pressure increases slowly and becomes significant as the value of $L$ decreasing; (2) in the case of air incompressibility, damping pressure always increases as the value of $L$ decreasing; (3) in contrast, in the case of air compressibility, damping pressure increases first, but drops in some area later as the value of $L$ decreasing.

\subsection{Two-dimensional perforated plate}

Now a two-dimensional rectangular perforated plate is considered. The plate width is comparable with the
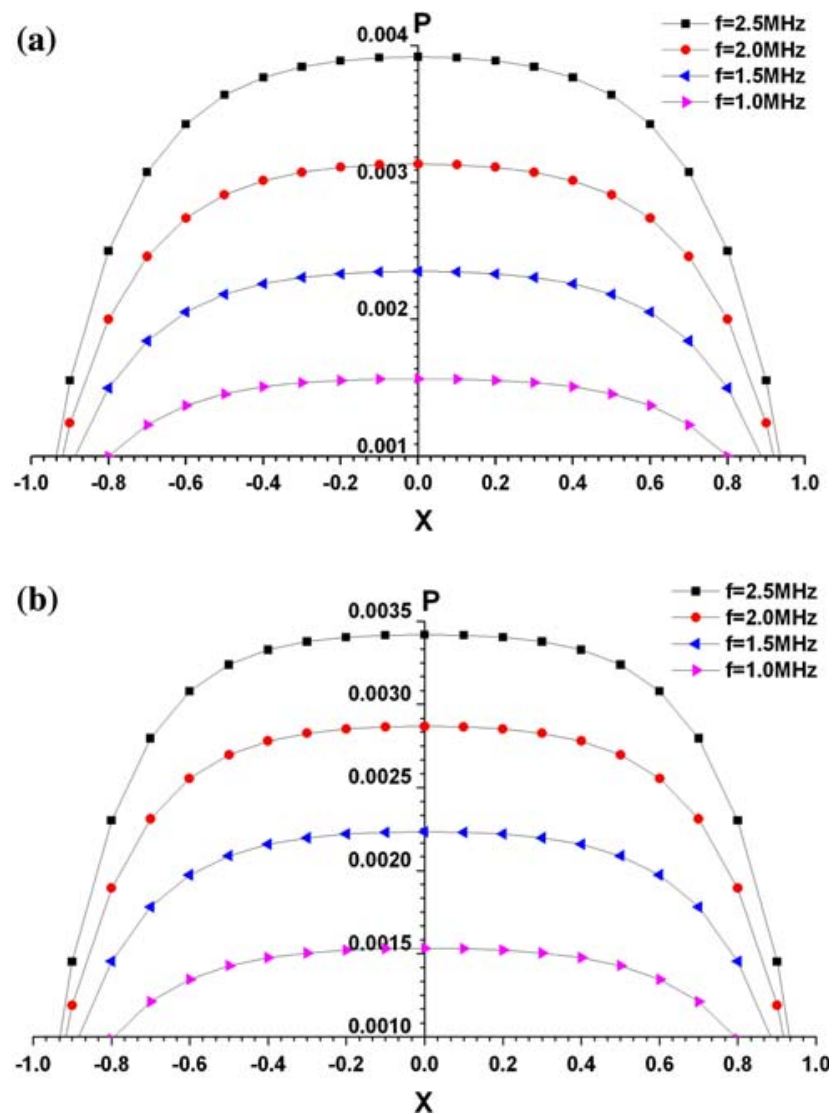

Fig. 2 The distributions of damping pressure under the perforated plate for different operating frequencies. a Damping pressure in the case of air incompressibility. b Damping pressure in the case of air compressibility

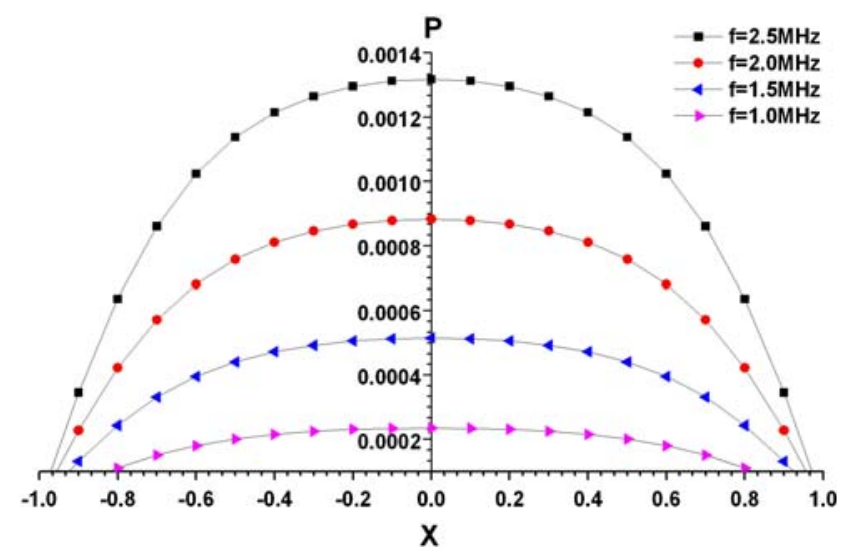

Fig. 3 The distributions of spring pressure under the perforated plate for different operating frequencies

length, thus the pressure caused by squeeze-film effects is a function of not only $X$ but also $Y$. Then Eq. 19 is

$\nabla^{2} \Psi_{0}(X, Y)-\left(L^{2}-j \sigma\right) \Psi_{0}(X, Y)=-L^{2} \varepsilon_{0}$. 


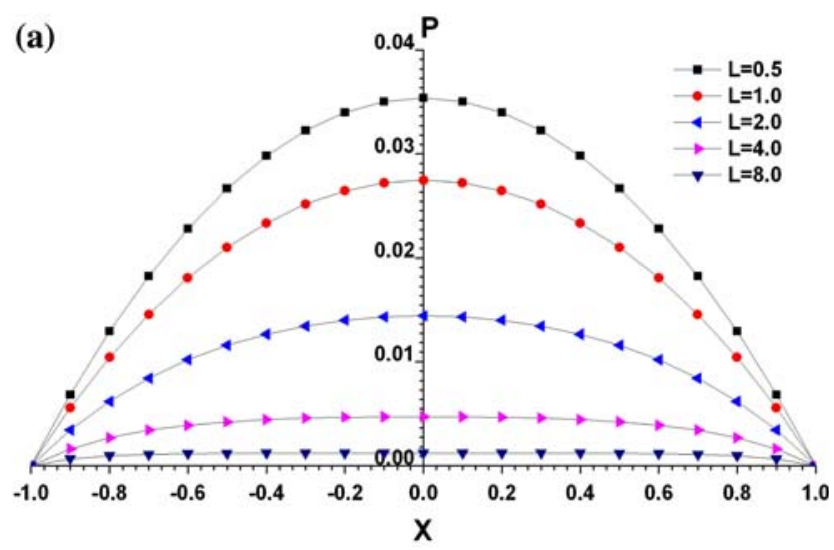

(b)

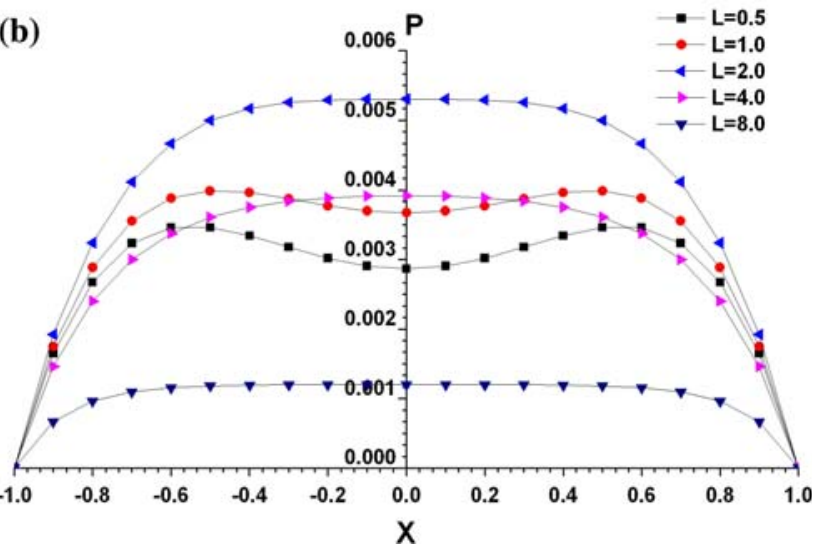

Fig. 4 The distributions of pressure under the perforated plate for different values of $L$. a Damping pressure in the case of air incompressibility. b Damping pressure in the case of air incompressibility

By adding a modified term to the one-dimensional solution, the two-dimensional solution can be written as (Myint 1973)

$\Psi_{0}(X, Y)=\Psi_{1}(X)+\varepsilon_{0}+\Psi_{2}(X, Y)$.

Substituting Eq. 30 into Eq. 29 and using boundary conditions, the equivalent form of Eq. 29 is

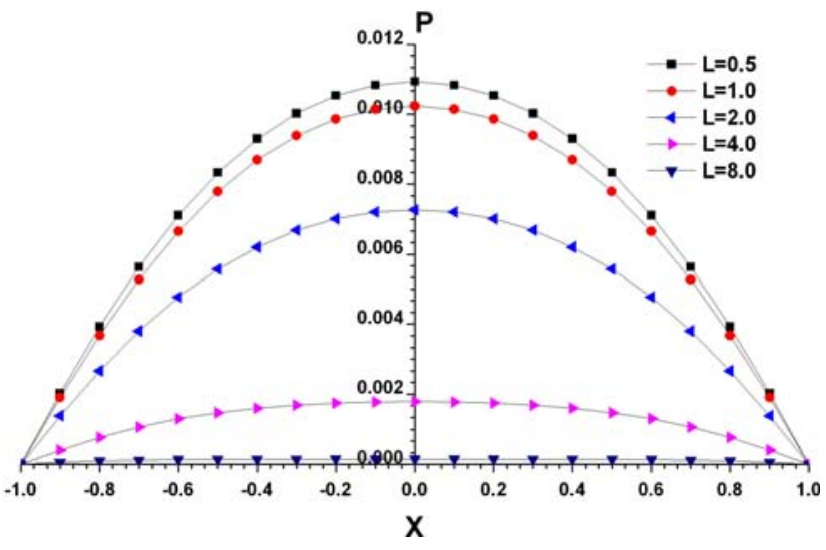

Fig. 5 The distributions of spring pressure under the perforated plate for different values of $L$

$\nabla^{2} \Psi_{2}(X, Y)-\left(L^{2}-j \sigma\right) \Psi_{2}(X, Y)=0$

with corresponding boundary conditions

$\Psi_{2}( \pm 1, Y)=0, \quad \Psi_{2}\left(X, \pm A_{R}\right)=\varepsilon_{0}-\Psi_{1}(X)$.

By separating variables and eigenfunction expansion methods, the solution of Eq. 31 is found to be

$\Psi_{2}(X, Y)=\Psi_{2}(X, Y)_{\mathrm{R}}+j \Psi_{2}(X, Y)_{\mathrm{I}}$,

where

$$
\begin{aligned}
\Psi_{2}(X, Y)_{\mathrm{R}}= & \frac{4 \sigma \varepsilon_{0}}{\pi} \sum_{n=0}^{\infty} \frac{(-1)^{n}}{(2 n+1)\left(L^{\prime 4}+\sigma^{2}\right)} \\
& \times\left(A_{2} \sigma+B_{2} L^{\prime 2}\right) \cos \frac{(2 n+1) \pi X}{2},
\end{aligned}
$$

$$
\begin{aligned}
\Psi_{2}(X, Y)_{\mathrm{I}}= & \frac{4 \sigma \varepsilon_{0}}{\pi} \sum_{n=0}^{\infty} \frac{(-1)^{n}}{(2 n+1)\left(L^{\prime 4}+\sigma^{2}\right)} \\
& \times\left(B_{2} \sigma-A_{2} L^{\prime 2}\right) \cos \frac{(2 n+1) \pi X}{2},
\end{aligned}
$$

and

$$
\left\{\begin{array}{l}
A_{2}=C_{2} \cdot\left[M_{2} \operatorname{ch}\left(K_{2} Y\right) \cos \left(\sigma Y / 2 K_{2}\right)+N_{2} \operatorname{sh}\left(K_{2} Y\right) \sin \left(\sigma Y / 2 K_{2}\right)\right] \\
B_{2}=C_{2} \cdot\left[N_{2} \operatorname{ch}\left(K_{2} Y\right) \cos \left(\sigma Y / 2 K_{2}\right)-M_{2} \operatorname{sh}\left(K_{2} Y\right) \sin \left(\sigma Y / 2 K_{2}\right)\right] \\
C_{2}=\frac{2}{\operatorname{ch}\left(2 K_{2} A_{R}\right)+\cos \left(\sigma A_{R} / K_{2}\right)}, K_{2}=L^{\prime} \sqrt{\frac{1+\sqrt{1+\left(\sigma / L^{\prime 2}\right)^{2}}}{2}} \\
L^{\prime 2}=L^{2}+(2 n+1)^{2} \pi^{2} / 4 \\
M_{2}=\operatorname{ch}\left(K_{2} A_{R}\right) \cos \left(\sigma A_{R} / 2 K_{2}\right), N_{2}=\operatorname{sh}\left(K_{2} A_{R}\right) \sin \left(\sigma A_{R} / 2 K_{2}\right)
\end{array} .\right.
$$


The pressure caused by squeeze-film effects of twodimensional perforated plate is

$\overline{\mathrm{P}}_{0}(X, Y)=\overline{\mathrm{P}}_{0}(X, Y)_{\mathrm{S}}+j \overline{\mathrm{P}}_{0}(X, Y)_{\mathrm{D}}$

where

$\overline{\mathrm{P}}_{0}(X, Y)_{\mathrm{S}}=\Psi_{2}(X, Y)_{\mathrm{R}}+\overline{\mathrm{P}}_{1}(X)_{\mathrm{S}}$,

and

$\overline{\mathrm{P}}_{0}(X, Y)_{\mathrm{D}}=\Psi_{2}(X, Y)_{\mathrm{I}}+\overline{\mathrm{P}}_{1}(X)_{\mathrm{D}}$.

So the spring force $F_{\mathrm{S}}$ and damping force $F_{\mathrm{D}}$ are, respectively,

$$
\begin{aligned}
F_{\mathrm{S}}= & \int_{-A_{R}}^{A_{R}} \int_{-1}^{1} \overline{\mathrm{P}}_{0}(X, Y)_{\mathrm{S}} \mathrm{d} X \mathrm{~d} Y \\
= & 4 \sigma \varepsilon_{0}\left[\frac{\left(\int_{0}^{1} A_{1} \mathrm{~d} Y-1\right) \sigma+L^{2} \int_{0}^{1} B_{1} \mathrm{~d} Y}{L^{2}+\sigma^{2}} A_{R}\right. \\
& \left.+\frac{8}{\pi^{2}} \sum_{n=1,3,5, \ldots}^{\infty} \frac{\sigma \int_{0}^{A_{R}} A_{2} \mathrm{~d} Y+L^{\prime 2} \int_{0}^{A_{R}} B_{2} \mathrm{~d} Y}{n^{2}\left(L^{\prime}+\sigma^{2}\right)}\right]
\end{aligned}
$$

and

$$
\begin{aligned}
F_{\mathrm{D}}= & \int_{-A_{R}}^{A_{R}} \int_{-1}^{1} \overline{\mathrm{P}}_{0}(X, Y)_{\mathrm{D}} \mathrm{d} X \mathrm{~d} Y \\
= & 4 \sigma \varepsilon_{0}\left[\frac{\sigma \int_{0}^{1} B_{1} \mathrm{~d} Y-\left(\int_{0}^{1} A_{1} \mathrm{~d} Y-1\right) L^{2}}{L^{2}+\sigma^{2}} A_{R}\right. \\
& \left.+\frac{8}{\pi^{2}} \sum_{n=1,3,5, \ldots}^{\infty} \frac{\sigma \int_{0}^{A_{R}} B_{2} \mathrm{~d} Y-L^{\prime 2} \int_{0}^{A_{R}} A_{2} \mathrm{~d} Y}{n^{2}\left(L^{\prime 4}+\sigma^{2}\right)}\right]
\end{aligned}
$$

For low operating frequency or high value of $L$, using the condition $\sigma / L^{2}, \sigma / L^{\prime 2} \rightarrow 0$, spring force $F_{\mathrm{S}}$ tends to be zero and damping force $F_{\mathrm{D}}$ can be simplified to the dimensionless form of the expression by $\mathrm{Bao}$ et al. (2003) exactly.

\section{Comparisons between theoretical analysis and ANSYS simulation}

According to the Table 1 by Mohite et al. (2005) and for the comparisons between different analytical results and ANSYS simulation results, a typical onedimensional rectangular perforated plate with square holes is considered. The plate thickness $h=5 \mu \mathrm{m}$ and the initial air gap $d_{0}=1 \mu \mathrm{m}$. The ratio of inner side to outer side of the divided pressure cell is set to be constant.

Figure 6 shows the comparisons between analytical results and the results by ANSYS simulation. The marks show the simulation results. The broken lines and the solid lines show the analytical results by Bao and by this paper, respectively.

Seen from Fig. 6, it is apparent that the analytical results without considering air compressibility overestimate damping effect. The analytical results by this paper agree with ANSYS simulation results better than that by Bao. However, for high operating frequency and high value of $L$, discrepancy between the analytical results by this paper and the simulation results becomes larger and even intolerable. So it is necessary to have analysis and give reasons for the large existing discrepancy. Besides the reasons mentioned in previous paper by Bao et al., some other possible reasons are: (1) while deriving the average penetrating rate, the expression of the horizontal air flow causing pressure difference, $p_{h}$, for one pressure cell is not very accurate
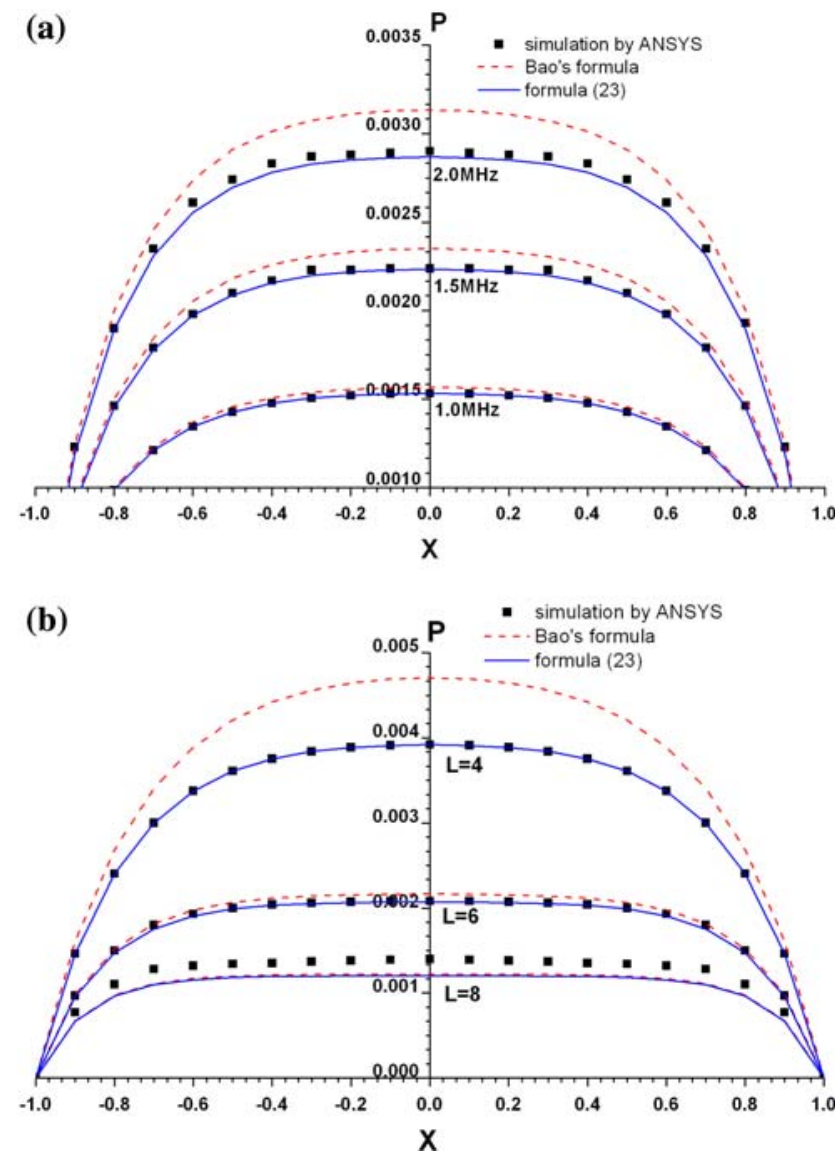

Fig. 6 Comparisons between analytical results and ANSYS simulation results. a Damping pressure with fixed value of $L$ $(L=5)$ for different operating frequencies. b Damping pressure with fixed operating frequencies ( $\mathrm{f}=2 \mathrm{MHz}$ ) for different values of $L$ 
when operating frequency is high; (2) for higher operating frequency, inertial effects become significant, and the pressure caused by squeeze-film effects is no longer infinitesimal compared to the ambient pressure. So inertial effects neglecting and the linearization to MRE are not very strict; (3) high value of $L$ means large holes for a fixed plate, so the assumption, which is made while dividing the plate into cells that the cell is much smaller than the plate is not very reasonable.

Besides comparisons between analytical results and ANSYS simulation results for damping pressure, comparisons for spring pressure are also made. It is found that ANSYS simulation results are a little smaller but still agree well with the analytical results for moderate operating frequency and low value of $L$.

\section{Conclusions}

Squeeze-film effects of perforated plates for low value of Reynolds number are analyzed through MRE, which reckons in most influential factors. It is found that the assumption that air is incompressible is no longer reasonable for high operating frequency and low value of $L$. And the reasons are not only because of the rapid growing spring pressure but also because of the considerable discrepancy between the cases of air incompressibility and compressibility for damping pressure. For the case of air incompressibility, damping pressure always increases as operating frequency increasing or the value of $L$ decreasing, but in the case of air compressibility, damping pressure increases first and then drops in some area later. So for low value of $L$ and high operating frequency, air compressibility should be considered.

By comparisons, the analytical results agree well with ANSYS simulation results. So the analytical analysis by this paper can be used to estimate the squeeze-film causing damping and stiffness added to the system. However, for more accurate analysis, a model considering inertial effects, nonlinear effects caused by large vibrating amplitude, and even slip velocity conditions at plates' boundaries has to be developed.

Acknowledgments This work was supported by the Distinguished Young Scholar Fund of National Natural Science Foundation of China (NSFC, Grant No. 10225209), key project from Chinese Academy of Sciences (Grant No. KJCX-SW-L2).

\section{Appendix A. Nomenclatures}

$$
\begin{array}{ll}
a & \text { plate half width } \\
b & \text { plate half length } \\
d_{0} & \text { initial air gap }
\end{array}
$$

$h \quad$ plate thickness

$h_{\text {eff }}$ effective plate thickness

$l \quad$ attenuation length

$p \quad$ squeeze-film pressure

$p_{\mathrm{a}}$ ambient pressure

$p_{\mathrm{h}} \quad$ pressure caused by horizontal air flow

$p_{\mathrm{v}}$ pressure caused by vertical air flow

$A_{R}$ aspect ratio

$C$ damping coefficient

$P_{\mathrm{D}}$ dimensionless damping force

$P_{\mathrm{S}}$ dimensionless spring force

$K$ stiffness coefficient

$K_{\mathrm{n}} \quad$ Knudsen number

$\bar{P}_{\mathrm{D}}$ dimensionless damping pressure

$\bar{P}_{\mathrm{S}} \quad$ dimensionless spring pressure

$Q_{z}$ penetrating rate

$R_{\mathrm{i}} \quad$ inner radius of the pressure cell

$R_{\mathrm{O}} \quad$ outer radius of the pressure cell

$\beta \quad$ inner radius to outer radius ratio

$\varepsilon_{0} \quad$ dimensionless vibration amplitude

$\mu \quad$ air viscosity coefficient

$\mu_{\text {eff }}$ effective air viscosity coefficient

$\rho$ air density

$\sigma \quad$ squeeze number

$\omega \quad$ operating frequency in radians per second

\section{References}

Andrews M, Harris I, Turner G (1993) A comparison of squeeze film theory with measurements on a microstructure. Sens Actuators A 36:79-87

Bao MH (2000) Micro mechanical transducers-pressure sensors, accelerometers, and gyroscopes. Elsevier, Amsterdam

Bao MH, Yang H, Sun YC, French PJ (2003) Modified Reynolds' equation and analytical analysis of squeeze-film air damping of perforated structures. J Micromech Microeng 13:795-800

Davies B, Montague S, Smith J, Lenkin M (1997) Micromechanical structures and microelectronics for acceleration sensing. Proc SPIE 3223:237-244

Fukui S, Kaneko R (1988) Analysis of ultra-thin gas film lubrication based on linearized Boltzmann equation: first report-derivation of a generalized lubrication equation including thermal creep flow. J Tribol Trans ASME 110:253-262

Gabrielson T (1993) Mechanical-thermal noise in micromechined acoustic and vibration sensors. IEEE Trans Electron Devices 40:903-909

Homentcovschi D, Miles RN (2004) Viscous damping of perforated planar micromechanical structures. Sens Actuators A 119:544-552

Kim E, Cho Y, Kim M (1999) Effect of holes and edges on the squeeze film damping of perforated micromechanical structures. Twealth IEEE international conference on micro electro mechanical systems (MEMS' 99) 296-301

Mohite SS, Kesari H, Sonti VR, Pratap R (2005) Analytical solutions for the stiffness and damping coefficients of 
squeeze-films in MEMS devices with perforated back plates. J Micromech Microeng 15:2083-2092

Myint.U T (1973) Partial differential equations of mathematical physics. Elsevier, New York

Schrag G, Wachutka G (2004) Accurate system-level damping model for highly perforated micromechanical devices. Sens Actuators A 111:222-228

Sharipov F, Seleznev V (1997) Data on inertial rarefied gas flows. J Phys Chem Ref Data 27:656-706

Sharipov F (1999) Rarefied gas flow through a long rectangular channel. J Vac Sci Technol A 17:3062-3066

Škov Z (1967) On acoustical resistance due to viscous losses in the air gap of electrostatic transducers. Acoustica 19:295297

Starr JB (1996) Squeeze-film damping in solid state accelerometers. Proc IEEE Solid State Sensors Workshop (Hilton Head Island, SC, USA, Jun 4-7) 44-47
Veijola T, Mattila T (2001) Compact squeezed-film damping model for perforated surface. Proc Trans'01 (München, Germany, Jun 10-14) 1506-1509

Veijola T, Ryhaanen T, Kuisma H, Lahdenperä J (2001) Circuit simulation model of gas damping in microstructures with non-trivial geometries. Proc Trans'01 (München, Germany, Jun 10-14)

Yan D, Lal A (2006) The squeeze film damping effect of perforated microscanners: modeling and characterization. Smart Mater Struct 15:480-484

Yang YJ, Gretillat MA, Senturia SD (1997) Effects of air damping on the dynamics of nonuniform deformations of microstructures. Int Conf on Solid State Sens Actuators. Transducers'97 (Chicago) 1093-1096 